\title{
Freeway Travel Time Prediction Research Based on A Deep Learning Approach
}

\author{
Junfeng Zhang ${ }^{1,2, a}$, Hongxi Chen ${ }^{1,2, b}$, Hong Zhou $^{3, c}$, Zhihai Wu ${ }^{1,2, \mathrm{~d}}$
}

\author{
${ }^{1}$ School of Transportation Science and Engineering, Beihang University, Beijing Key Laboratory for \\ Cooperative Vehicle Infrastructure Systems and Safety Control, 37 Xueyuan Road, Beijing, China \\ ${ }^{2}$ Jiangsu Province Collaborative Innovation ${ }^{2}$ Jiangsu Province Collaborative Innovation Center of \\ Modern Urban Traffic Technologies, SiPaiLou \#2, Nanjing, China \\ 3 Jiangsu Communications Holdings Co. Ltd. Jiangsu 210002,China; \\ abothwellzhang@163.com, b1208403152@qq.com, ck kgyyb@126.com
}

Keywords: Deep learning, Toll data, Travel time prediction, Automatic encoder

\begin{abstract}
Accurate prediction of freeway travel time is quite significant for traffic management and travelers' trip decisions. A model of stacked automatic encodersforfreeway travel time prediction is proposed. First, obtain the hourly average travel time by processingfreeway toll data. Considering data quantity and travel time scheduling, a travel timeprediction model based on stacked automatic encodersis established then. The automatic encoder is trained with unsupervised learning step by stepby using historical travel time data, adjust the parameters of prediction layer with gradient descent method.Ultimately the one-hour travel time is predicted using the past three-hours' average travel time. Compared to the traditional BP neural network prediction model, the Root Mean Square Error of this methoddrops by $13.6 \%$,verifying the validity of the model.
\end{abstract}

\section{Introduction}

In recent years, due to rapid economic development and the increasing vehiclesinventory, traffic congestion increment is becoming evident, reducing freeway efficiency as well as bringing a lot of inconvenience to travelers. Freeway travel time prediction can provide data reference for freeway analysis andmanagement as well as traveler's arrangement.

Road travel time prediction research had been conducted throughout the world. Time series methods had been presented by Anderson to explore the travel time ${ }^{[1]}$. A queuing prediction model had been presented by Doh without consideringtraffic controls and other vehicles' influence ${ }^{[2]}$. A pattern matching model had been established by Kana to predict the road travel time ${ }^{[3]}$. Time series and linear methods had been integrated together by John to forecast travel time ${ }^{[4]}$. A better prediction model had been found by Dion for the nonlinear traffic flow ${ }^{[5]}$. Mainland travel time researches had been conducted by scholar Yang Zhaosheng using all kinds of methods, and therefore synthesis algorithm integrating a variety of methods had been established ${ }^{[6-9]}$. A result that the accuracy decrement of regression algorithm had been found by Huang Xuefeng ${ }^{[10]}$. Another result that difference of prediction results was of existence in different cases of the very prediction model had been found by Li Huibing ${ }^{[11]}$. From a macro perspective, study and research of travel time prediction had been implemented earlier abroad, and many models had been applied in practical applications. In domestic field, attention is mainly focused onthe simulation research of urban arterials and expressway, and little attention on freeway travel time. Based on the deep learning approach and freeway toll data, a stacked automatic encoder model of freeway travel time prediction is established in this paper, and improves the prediction effectivenesscompared with the 
common method of BP neural network.

\section{Data Preprocessing}

The data is offered by the South Guangzhou airport freeway, which is an open data resources from the Open ITS. The toll data consists of ten days' data and extracts 10 samples with a five minutes interval. Because toll stations are sparsely distributed in the freeway, the toll data is of low spatial coverage. To solve this problem, the data need to be preprocessed. Steps are: deletion of abnormal data, and gain of data vectors.

\subsection{Deletion of Abnormal Data}

Due to the various external and internal factors in freeway system, anthropogenic factors such as car crashes and drivers side resting, and non-anthropogenic factors such as snow and rain, therefore, it is necessary to eliminate the abnormal data before upcoming process. The method is eliminate $\mathrm{T}$ while $\mathrm{T} \gg>\mathrm{T}_{0}$ (where $\mathrm{T}$ is the travel time originating from toll data and $\mathrm{T}_{0}$ is the normal travel time). It is shown in Figure 1, data before processing are more discrete, after processing the dispersion of the data had been greatly improved.

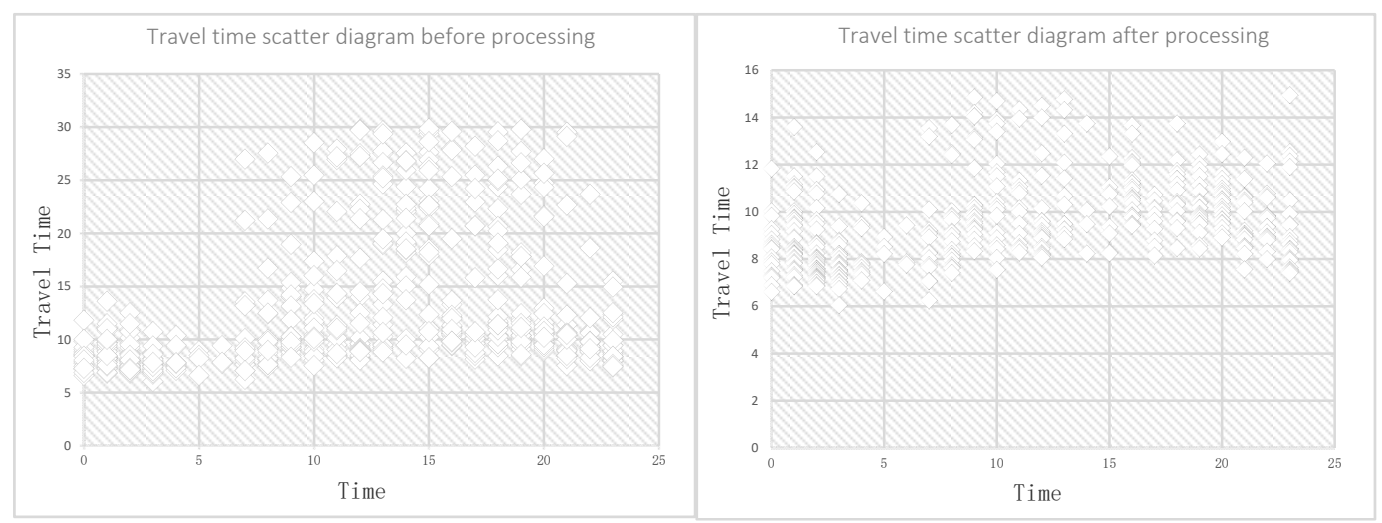

Fig. 1 Travel time scatter diagram before and after processing

\subsection{Data vector gain}

After deleting the abnormal data, the amount of data is still not enough, accounting for the necessity of the training data's abundance increment with some processing methods. Then the training of the neural networks would be provided for multiple layers of data input, which is of advantage for the model convergence. The approach adopted by the paper is input the historical travel timewith dislocation order. The result was shown at table 1 and table 2 .

Table 1 Average Travel Time of the advanced 7days before processing

\begin{tabular}{ccccccccc}
\hline Time & 1 & 2 & 3 & 4 & 5 & 6 & 7 & 8 \\
\hline Aver time & 8.39 & 8.89 & 8.21 & 7.80 & 8.14 & 8.07 & 8.35 & 8.90 \\
\hline
\end{tabular}

Table 2Average Travel Time of the advanced 7days after processing

\begin{tabular}{ccccccccc}
\hline Time & $1-3$ & $2-4$ & $3-5$ & $4-6$ & $5-7$ & $6-8$ & $7-9$ & $8-10$ \\
\hline & 8.39 & 8.89 & 8.21 & 7.80 & 8.14 & 8.07 & 8.35 & 8.90 \\
Aver time & 8.89 & 8.21 & 7.80 & 8.14 & 8.07 & 8.35 & 8.90 & 9.25 \\
& 8.21 & 7.80 & 8.14 & 8.07 & 8.35 & 8.90 & 9.25 & 10.74 \\
\hline
\end{tabular}




\section{Travel Time Prediction Model}

\subsection{Model Determination}

There are many common models and methods of deep learning approach, such as stacked automatic encoders (SAEs), restricted Boltzmann machines (RBM), deep belief networks (DBN), convolutional neural networks (CNN). Though deep neural network is more power than shallow neural networks in model learning, but typically only one hidden layer is contained in the present travel time prediction method, training with traditional training methods may have many problems. Therefore, stacked automatic encoder is chosen for building models for travel time prediction in this paper, namely heap automatic encoder travel time prediction model.

\subsubsection{Automatic encoder}

Stacked automatic encoder is a deep networks construction composed of several simple automatic encoders or sparse-depth network encoders. In this paper, choose the former ones contributes to the construction of the travel time prediction modal based on SAEs.

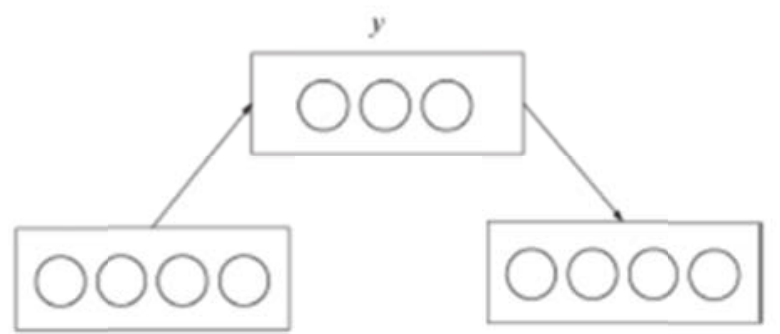

Fig. 2Automatic Encoder

The simplest automatic encoder is illustrated in Figure 2, which has only one hidden layer and whose hidden layer's unit number is set manually. Given a set of training samples $\{x(1), x(2), x(3), \ldots\}$, where $x(i) \in R d, x(i)$ means the average hourly travel time of first three hours $t_{n-3}, t_{n-2}, t_{n-1}$. The first input $x(i)$ would be expressed as $y(x(i))$ based on equation (1),and then expressed as $z(x(i))$ based on equation (2).

$y(x)=f\left(w_{1} x+b\right)$

$z(x)=g\left(w_{2} y(x)+c\right)$

Where, $W_{1}$ means the weights matrix connecting the input layer and the first hidden layer. bis an encoding errors vector. $W_{2}$ is a decoding matrix connecting the first hidden layer and the second hidden layer. $\mathrm{c}$ is a decoding error vector. $f(x)$ and $g(x)$ are expressed by the function $1 /(1+\exp (-x))$. In order to minimize the rebuilding error $L(X, Z)$, model parameter $\theta$ is needed. $\theta=\arg \min L(X, Z)=\frac{1}{2} \sum_{1}^{N}\left\|x^{(i)}-z\left(x^{(i)}\right)\right\|^{2}$

After Network's trained, when input $x(i)$, namely the first three hours of the test sample data, hidden layer's encoding will be conducted to $x(i)$ in encoding networks, then rebuilding the $x(i)$ by decoding from hidden layer.

3.1.2Stacked automatic encoder travel time prediction model

Stacked travel time prediction model is a deep network formed by the superposition of automatic encoders, whose current layer encoder's output data will be sent to the next layer as input data. 


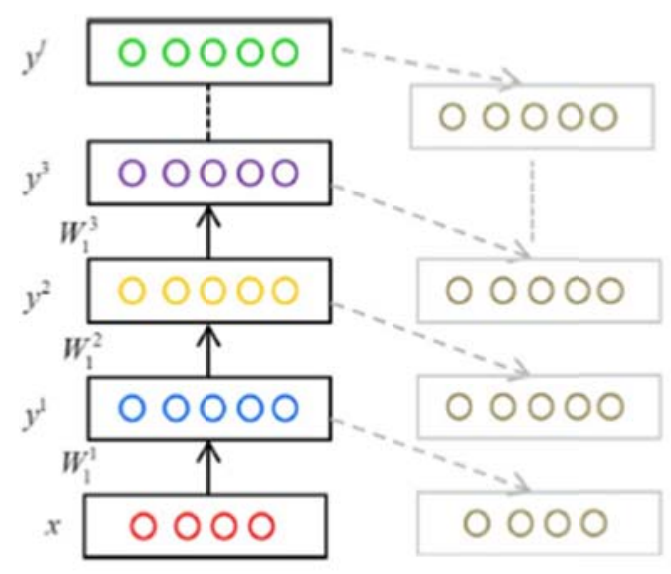

Fig. 3Stacked Automatic Encoders Model Structure Diagram

For the first layer, unsupervised learning is conducted to the automatic encoder's training by using the travel time of first 7days, resulting in the $W_{1}$ and $b_{1}$ meeting the accuracy requirements. Then the output data would be sent to the next automatic encoder and proceed the same training. After the training of all automatic encoders is completed, the final output namely $W_{n}$ and $b_{n}$ will be the input of the prediction layer and the parameters of the prediction layer will be adjusted by the deviation between predicted and actual data, contributing to the construction of the travel time prediction modal based on SAEs.

3.2 Training Method

After the unsupervised training of the hidden layers, use gradient descent method to adjust parameters from the logistic regression layer of the prediction layer. Training process is based on the method proposed by G.E.Hinton, S.Osindero, and Y.-W. Teh ${ }^{[12]}$, as well as Y. Bengio, P. Lamblin. D. Popovici and H. Larochelle ${ }^{[13]}$.

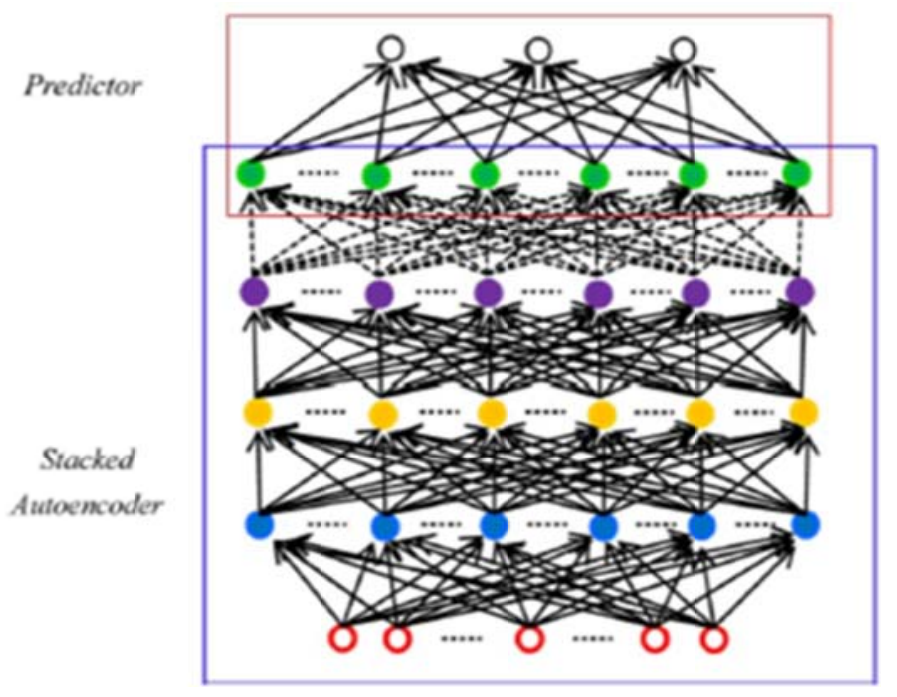

Fig. 4 SAEs Prediction Model Diagram

Algorithm is as follows:

For given training sample $\boldsymbol{x}$ (first three hours' average hourly prediction time, $t_{n-3}, t_{n-2}, t_{n-1}$.) and the required amount of hidden layers.

Step 1) Training SAEs

--Set the sparse weight $\gamma$, the sparse parameter $\rho$, randomly initialize weight matrix and deviation vector.

--Train the hidden layer layer by layer.

--Use the k-hidden layer's output as the $(\mathrm{k}+1)$ hidden layer's input. The first hidden layer's input is 
the training set.

--Minimize the objective function to encode the $(\mathrm{k}+1)$ hidden-layer's parameters

Step 2) Fine-tuning the entire network

--Randomly initialize $\left\{W_{1}, b_{1}\right\}$ or supervise the training.

--Change the technical parameters of the prediction layer based on gradient optimization using BP method.

\section{Travel Time Prediction}

\subsection{Design of Experiment Parameters}

In SAEs, number of input and hidden layers, number of hidden units of each hidden layer and the input data have impact on freeway travel time prediction. So this example is divided into three cases: (1)SAEs model with same number of hidden layer and different number of hidden units. Compare and analyze the travel time prediction effect of each case. (2)SAEs model with same number of hidden units and different number of hidden layers Compare and analyze the travel time prediction effect of each case.(3) Compare and analyze the effect of traditional BP algorithm with only one hidden layer and the proposed SAEs prediction model.

\subsection{Evaluation}

In order to assess the validity of the model, two performance indicators are used, which are defined as:

Mean Average Percentage Error: MAPE $=\frac{1}{n} \sum_{i=1}^{n} \frac{\left|f_{i}-\hat{f_{i}}\right|}{f_{i}}$ (4)

Root Mean Square Error: $R M S E=\left[\frac{1}{n} \sum_{i=1}^{n}\left(\left|f_{i}-\hat{f}_{i}\right|\right)^{2}\right]^{\frac{1}{2}}$

Where $f$ is real value and $\hat{f}$ is prediction value.

\subsection{Example}

Set 2 hidden layers for the model, with different number of hidden units namely 10, 50, 100, the result is as follows:

\begin{tabular}{ccc}
\multicolumn{3}{c}{ Table 3Result of Different Hidden Layer Units } \\
\hline $\begin{array}{c}\text { Number of Hidden } \\
\text { layer units }\end{array}$ & MAPE & RMSE \\
\hline 10,10 & 0.0901 & 1.0673 \\
50,50 & 0.0880 & 1.0420 \\
100,100 & 0.0964 & 1.1735
\end{tabular}

It is obvious that when the number of hidden layer units is 50 , 50 , the model has better evaluations performance than the other two cases, showing that the number of hidden layer units has impact on the model prediction accuracy.

Set 10 units for each hidden layer, with different number of hidden layers namely 2, 3, 5, the result is as follows: 
Table 4Result of different Hidden Layer

\begin{tabular}{ccc}
\hline $\begin{array}{c}\text { Number of Hidden } \\
\text { layer }\end{array}$ & MAPE & RMSE \\
\hline 2 & 0.0901 & 1.0673 \\
3 & 0.0895 & 1.0589 \\
5 & 0.0880 & 1.0464 \\
\hline
\end{tabular}

In this case, when the hidden layer is 5, the model has better evaluations performance than the other two cases, showing that number of the hidden layer has impact on the model prediction accuracy. Within a certain range, with the increase of hidden layers, the prediction accuracy will increase.

Finally, use BP algorithm to obtain the evaluation of travel time prediction. Together with evaluation obtained from above, the result is as shown in Table 5.

Table 5Result of all evaluations

\begin{tabular}{cccc}
\hline Evaluation & & MAPE & RMSE \\
\hline BP network & & 0.0959 & 1.2059 \\
Hidden layer units' number & 10,10 & 0.0901 & 1.0673 \\
(layer number is 2) & 50,50 & 0.0880 & 1.0420 \\
Hidden layer number & 100,100 & 0.0964 & 1.1735 \\
\cline { 2 - 4 } (units number is10) & 2 & 0.0901 & 1.0673 \\
& 3 & 0.0895 & 1.0589 \\
& 5 & 0.0880 & 1.0464
\end{tabular}

When using the same data, the evaluations performance of SAEs is better than the BP neural networks', showing that the SAEs model is endowed with more accuracy and stability in freeway travel time prediction.

\section{Summary}

A SAEs model for freeway travel time prediction was put forward in this paper. First of all, the hourly average travel time was acquired by dealing with the freeway toll collection data. And the first 7-days'data was set to training data while the last 3-days'data experimenting data. Then SAEs model for travel time prediction was established in considering the data quantity and travel time scheduling. Using historical travel time data, the automatic encoder was trained with unsupervised learning step by step, the parameters of the prediction layer being adjusted by the mean of Minimum Reconstruction Error and Gradient Descent Method and ultimately the one-hour travel time being predicted using the forward three-hours' average travel time. Compared to the traditional BP neural network prediction model, the RMSE of the built stacked automatic encoders travel time prediction model dropped by $13.6 \%$ while the MAPE dropped by $8.6 \%$, verifying the validity of the model. 


\section{Acknowledgments}

This paper is one of the initial achievement of the sub-topics "Technology and systems development based on analysis of multi-scale road network running posture large data," which belongs to the national science and technology support program "Intelligent state highway network monitoring and security services to protect key technologies and system integration." (Program No. 2014BAG01B02)

\section{Reference}

[1]Wosyka Jakub, Pribyl Pavel. Real-time travel time estimation on highways using loop detector data and license plate recognition[C]/Proceedings of 9th International Conference, ELEKTRO. Slovakia: IEEE, 2012: 391-394.

[2]Nam Do Hdynamics and Donald R. Drew. Traffic Method for Estimation Freeway Travel Time in Real Time from Flow Measurements. Journal of transportation engineering.1996.(5).185-19.

[3] Otokita Tooru, Hashiba Kana, Oda Toshihiko. Travel Time Prediction Based on Pattern Extraction from Database [J]. Papers of Technical Meeting on Transportation and Electric Railway, 1999, 99(11/20):39^4.

[4] Francois Dion, Hesham Rakha. Estimating Spatial Travel Times using Automatic Vehicle Identification Data[C]the proceedings of the 82 annual meeting of the transportation research board. Washington D.C, 2003.

[5]Xiaoyan Zhang and John A. Rice. Short-term time travel Prediction. Transportation Research[J] PartC.2003:187- 210.

[6]Yang Zhaosheng.Study on Synthetic Link Travel Time Prediction Model of Key Theory of ITS [J]. Journal of Traffic and Transportation Engineering 2001, 1 (1): 65-67.

[7]Zhu Zhong, Yang Zhaosheng.A Real-time Travel Time Estimation Model Based on the Kalman Filtering [J] Theory for Traffic Flow Guidance System,1999, 19 (9): 74-78.

[8] Yang Zhaosheng, Zhu Zhong. Real-time Travel Time EstimationModelBased on Back Propagation Neural Network[J]. SYSTEMS ENGINEERING-THEORY \& PRACTICE, 1998,19(8):59-64.

[9]Yang Zhaosheng,Bao Lixia,Zhu Guohua. An Urban Express Travel Time Prediction Model Based on Fuzzy Regression[J]. Journal of Highway and Transportation Research and Development, 2004, 21(3): 78-81.

[10]Huang Xuefeng,Zhou Jianfeng. Travel Time Prediction of Highway Vehicles[J].Highway, 2009, (2):116-120.

[11]Li Huibin,Yang Xiaoguang.Data Fusion Method for Accuracy Evaluation of Travel Time Forecast[J]. Journal of Tongji University (Natural Science)2013, 41 (1): 60-65.

[12] G. E. Hinton, S. Osindero, and Y.-W. Teh,A Fast Learning Algorithm for Deep Belief Nets, Neural Comput, vol. 18, no. 7, pp. 1527-1554, Jul. 2006.

[13] Y. Bengio, P. Lamblin, D. Popovici, and H. Larochelle, "Greedy layerwise training of deep networks," in Proc. Adv. NIPS, 2007, pp. 153-160. 